\title{
The Flavonoid Kurarinone Regulates Macrophage Functions via Aryl Hydrocarbon Receptor and Alleviates Intestinal Inflammation in Irritable Bowel Syndrome
}

\author{
Xiang $\mathrm{Xu}^{\mathrm{I}, *}$ \\ Qiwei Dong ${ }^{1, *}$ \\ Qingling Zhong ${ }^{2}$ \\ Wenbo Xiu ${ }^{1,2}$ \\ Qinyuan Chen ${ }^{1,2}$ \\ Jinxia Wang' \\ Zhou Zhou ${ }^{1,2}$
}

'Clinical Immunology Translational Medicine Key Laboratory of Sichuan Province, Sichuan Provincial People's Hospital, University of Electronic Science and Technology of China, Chengdu, People's Republic of China; ${ }^{2}$ Department of Gastroenterology and Hepatology, Sichuan Provincial People's Hospital, University of Electronic Science and Technology of China, Chengdu, People's Republic of China

*These authors contributed equally to this work
Correspondence: Zhou Zhou

Clinical Immunology Translational Medicine Key Laboratory of Sichuan Province, Sichuan Provincial People's Hospital, University of Electronic Science and Technology of China, Chengdu,

People's Republic of China

Tel +86-028-8739420l

Fax +86-028-8739420 I

Email doublezhou2006@126.com
Background: Irritable bowel syndrome (IBS) is characterized with abdominal pain, bloating, and changes in bowel habits, and dealing with IBS is still a clinical challenge. The pathogenesis of IBS has been reported to be linked to low-grade mucosal inflammation, and macrophages contribute to the pathological process of this disease. Kurarinone (KAR), a flavanoid derived from Sophora flavescens, has been found medically effective in many inflammatory conditions and cancers. KAR was previously reported to inhibit LPS-induced expression of inflammatory cytokines in macrophages, whether and how KAR regulates the functions of macrophage in IBS remains to be elusive.

Methods: We established a TNBS-induced IBS mouse model, in which KAR was administrated, and mucosal cytokine expression was measured by qRT-PCR. Additionally, mouse macrophages were generated in vitro and their responses to LPS were evaluated by flow cytometry and qRTPCR. $\mathrm{AhR}^{+/+}$or $\mathrm{AhR}^{-/-}$macrophages were transferred into DTx-treated CD11b-DTR transgenic mice to investigate the role of AhR in IBS. We collected colonic biopsies and peripheral blood samples from 64 patients with IBS, and analyzed AhR expression by qRT-PCR.

Results: We found KAR effectively alleviated visceral hypersensitivity and maintained intestinal barrier functions in mice with IBS. KAR inhibited LPS-induced macrophage activation and expression of pro-inflammatory genes, while increased anti-inflammatory gene expression including IL-10 in an AhR-dependent manner. Using macrophage-depleted mice, we found that chimera mice with $\mathrm{AhR}^{-/}$macrophages were more susceptible to TNBS-induced IBS and the therapeutic effect of KAR on IBS was significantly impaired in mice with $\mathrm{AhR}^{-/-}$macrophages. Additionally, we found $\mathrm{AhR}$ expression in macrophages of IBS patients was associated with the disease severity.

Conclusion: Our findings provide new evidences that KAR regulates IBS development via macrophage-intrinsic AhR. KAR might show promise as an immunomodulatory therapeutic agent in treating IBS.

Keywords: aryl hydrocarbon receptor, irritable bowel syndrome, macrophage, kurarinone, flavonoid

\section{Introduction}

Irritable bowel syndrome (IBS) is a common condition affecting the digestive system, characterized with abdominal pain, bloating, and changes in bowel habits. The prevalence rate of patients with IBS in China who meet the Rome III diagnostic criteria has reached $12.9 \%$, which has become one of the common diseases of the 
digestive tract. ${ }^{1}$ Therefore, dealing with IBS is still an important clinical challenge. Although the exact etiology and pathogenesis of IBS are still unclear, accumulating evidences also show that dysregulated gut microbiota and imbalanced mucosal immunity are critical factors in the pathogenesis of IBS. $^{2}$

IBS has been reported to be linked to low-grade mucosal inflammation. ${ }^{3,4}$ About $25 \%$ of IBS patients had symptoms of gastroenteritis, dysentery and other infectious diseases. Mucosal inflammation in IBS patients is often observed, probably attributing to compromised epithelial barrier functions and the translocation of luminal bacteria, parasites or viruses, which might abnormally initiate immune responses in the lamina propria., ${ }^{2,5}$ The activation of mucosal immune system in IBS patients is significantly up-regulated, including increased immune cell infiltration and inflammatory cytokine expression. ${ }^{5}$ Intestinal mucosa biopsies from patients with IBS show an increased number of mast cells, T lymphocytes and other immune cells. ${ }^{6-8}$ In addition, there are a number of macrophages in the intestine, which play an important role in maintaining the microbial homeostasis on the surface of intestinal mucosa and regulating the continuous renewal of intestinal epithelial cells. ${ }^{9}$ Macrophages are not only an important part of innate immunity, but also participate in the development of adaptive immune cells, which they cooperate with to contribute to the pathological process of intestinal inflammation, such as inflammatory bowel disease (IBD) and IBS. ${ }^{10}$ However, the underlying pathogenesis of these changes in immune responses is still largely unclear.

Kurarinone (KAR), a flavonoid derived from Sophora flavescens, has been reported to be medically effective in many inflammatory conditions and cancers. ${ }^{11-13}$ KAR exerts potent antioxidant and immunosuppressive functions via activating an array of antioxidant enzymes by abrogating the KEAP1 inhibition of Nrf2, which is wellknown as a key transcription factor in the antioxidant defense system. ${ }^{14}$ Moreover, KAR was found to inhibit the process of experimental autoimmune encephalomyelitis (EAE) via blocking Th1 and Th17 cell differentiation ${ }^{12}$ Although KAR was previously reported to inhibit LPSinduced expression of inflammatory cytokines in RAW264.7 cells (a macrophage cell line), ${ }^{14}$ whether and how KAR regulated the functions of macrophage in IBS remains to be elusive. In this study, we found KAR effectively alleviated visceral hypersensitivity and inflammatory responses in a post-inflammatory (PI)-IBS mouse model, and its regulation of the anti-inflammatory functions of macrophage via aryl hydrocarbon receptor (AhR) was indispensable for this therapeutic effect. Additionally, we found AhR expression in macrophages of IBS patients was closely associated with the disease severity. Our results shed new lights on the mechanisms of how KAR regulates macrophage functions, which might be a potential therapeutic drug for IBS.

\section{Materials and Methods}

\section{Patients}

A total of 64 patients with IBS diagnosed according to the Rome III criteria and 37 healthy controls were all recruited from the Department of Gastroenterology and Hepatology, Sichuan Provincial People's Hospital (Chengdu, China). Constipation-predominant IBS (IBS-C) was present in 21 patients $(5 / 21$ patients had a history of gastroenteritis), diarrhea-predominant IBS (IBS-D) in 26 patients $(3 / 26$ had suffered from gastroenteritis), and IBS with mixed bowel habits (IBS-M) in 17 patients. Colonic biopsies were collected during colonoscopy procedures, which were used for mRNA expression. For cell isolation, fresh peripheral blood samples were collected using EDTAanticoagulated blood collection tubes (BD green cap, $\mathrm{BD}$ Biosciences, San Diego, CA, USA). Blood sera were collected using serum separator tube (BD red cap, BD Biosciences) and cytokine measurement was performed as described previously. ${ }^{15}$ The study was conducted in accordance with the Declaration of Helsinki and approved by the Institutional Review Board for Clinical Research of the Sichuan Provincial People's Hospital. All subjects signed an informed consent before participation.

\section{Animal}

$\mathrm{AhR}^{ \pm}$mice and CD11b-DTR transgenic mice were obtained from The Jackson Laboratory (Bar Harbor, ME, USA). Wild type (WT) mice were purchased from the Shanghai Model Organisms (Shanghai, China). All mice enrolled in the current study were male and on a C57BL/ B6J background and used at their age of 8-10 weeks. All mice were maintained in our facility under specific pathogen-free (SPF) conditions. According to our recent report, ${ }^{16}$ macrophages were removed in CD11b-DTR transgenic mice using diphtheria toxin (DTx) and the removal efficiencies were verified by flow cytometry, showing over $75 \%$ of $\mathrm{CD}_{11 \mathrm{~b}^{+}}$macrophages were removed. All animal experiments in this study complied with the ARRIVE guidelines, and were approved by the 
Animal Care and Use Committee at Sichuan Provincial People's Hospital and performed in accordance with the National Institutes of Health guide for the care and use of Laboratory animals.

\section{Peritoneal Macrophage Isolation and Adoptive Transfer}

Mouse peritoneal cell suspension were prepared. After being sacrificed, mice were injected with $5 \mathrm{~mL}$ of PBS in the peritoneal cavity and the anterior and lateral walls of the abdomen were gently massaged for 5 minutes. Peritoneum washes were collected, which were then filtered through a nylon filter $(70 \mu \mathrm{m})$ and macrophages (F4/ $80^{+}$cells) were isolated from peritoneal cell suspension by using the Macrophage Isolation Kit (Peritoneum), an MS Column, and a MiniMACSTM Separator. Then, $\mathrm{AhR}^{+/+}$or $\mathrm{AhR}^{-/-}$macrophages $\left(2 \times 10^{6}\right.$ per recipient mice $)$ were transferred intravenously into CD11b-DTR transgenic mice, which had underwent macrophage depletion.

\section{IBS Mouse Model, Abdominal Withdrawal Reflex (AWR), and Visceromotor Response (VMR) Test}

Mice were received with a single enema of 2,4,6-trinitrobenzenesulfonic acid (TNBS) to induce colonic inflammation as described previously. ${ }^{15}$ Five weeks after TNBS insults, the AWR test was used to evaluate the visceral hypersensitivity in response to colorectal distention (CRD) in mice. ${ }^{17}$ Briefly, anesthetized mice were inserted intrarectally with a silicone balloon-urethral catheter and the balloon was distended at indicated volumes for 30 seconds every 4 minutes one hour after these mice revived. The AWR scoring standard was documented elsewhere. ${ }^{18}$ In addition, we also measured the abdominal electromyography activity to determine the visceral hypersensitivity. Briefly, CRD was performed at indicated volumes as described above, and then VMR was recorded by quantifying reflex contractions of the abdominal musculature. Two electrodes (diameter: $0.08 \mathrm{~mm}$ ) were implanted in the abdominal external oblique muscle and a third in the abdominal skin. Abdominal electromyography activity was recorded (amplification: 10,000×). The baseline of electromyographic activity was subtracted from that recorded during distension. All animal experiments were approved by the Animal Care and Use Committee at Sichuan Provincial People's Hospital.

\section{Colon Transportation Test (CTT) and Stool Consistency Evaluation}

The status of mouse intestinal motility was evaluated using CTT as reported elsewhere. ${ }^{18}$ Briefly, mice were administered intragastrically with active carbon in the stomach, and the time these mice took to defecate the first black stool was monitored. In addition, we collected the total stool within 8 hours and evaluated their consistency by Bristol stool grade. Normal shaped stool was defined as 1 point, soft or deformed stool as 2 points, and water-like stool as 3 points.

\section{Generation of Mouse Bone Marrow-Derived Macrophages (BMDM) and Human Monocyte-Derived Macrophages (MDM)}

Mouse bone marrow cells and human peripheral blood monocytes were obtained as described previously. ${ }^{16}$ For mouse BMDM generation, bone marrow cells were incubated with macrophage colony-stimulating factor (M-CSF, $10 \mathrm{ng} / \mathrm{mL}$ ) in the Iscove's Modified Dulbecco's Medium (IMDM) supplemented with 10\% fetal bovine serum (FBS). ${ }^{19}$ After 7 days of incubation, flow cytometry was performed to evaluate the formation of mature BMDM, and $>90 \%$ of these cells co-expressed CD11b and F4/80. For human MDM, purified peripheral monocytes were cultured in the presence of M-CSF (10 ng/mL) plus granulocyte-macrophage colony-stimulating factor (GM-CSF, $1 \mathrm{ng} / \mathrm{mL}$ ) in RPMI-1640 supplemented with 10\% FBS for 5 days. The formation evaluation was performed as described above. ${ }^{20}$

\section{Measurement of Intestinal Barrier Function}

Intestinal barrier function was determined as reported previously. ${ }^{16}$ After being starved overnight, mice were orally administrated with FITC-dextran $(2 \mathrm{mg} / 10 \mathrm{~g}$ body weight, Sigma-Aldrich, St. Louis, MO, USA) by gastric gavage 4 hours prior to sacrifice. Blood samples were obtained by cardiac puncture, and serum separator tubes (BD red cap) were used to collected mouse sera. The fluorescence intensity of FITC-dextran in sera was assessed (excitation: $485 \mathrm{~nm}$, emission: $525 \mathrm{~nm}$ ) using an Epoch 2 reader (BioTek, Winooski, VT). Serum LPS concentration was measured with a quantitative chromogenic 
limulus amoebocyte lysate (LAL) QCL-1000 test kit (LONZA), following the manufacturer's protocols.

\section{Quantitative Real-Time PCR (qRT-PCR)}

As reported previously, ${ }^{16}$ Trizol (TransGen Biotech, Beijing, China) was used to extracted total RNA from cells or tissues. Reversible transcription PCR was performed to transcribe the total RNA into cDNA by a reverse transcription kit (TransGen Biotech). A SYBR Green PCR kit (TaKaRa, Japan) was used to carried out qRT-PCR in a fluorescence thermocycler (Roche Diagnostics, Germany) and primers used in this study were listed in Supplementary Table 1. An Epoch 2 reader (BioTek, Winooski, VT) was used to acquire the data from qRT-PCR assays.

\section{Flow Cytometry}

Flow cytometric analysis of cells cultured in vitro and cells obtained from tissues was carried out as described previously. ${ }^{16}$ Briefly, intracellular cytokine staining was measured using the Mouse IL-10 Secretion AssayDetection Kits purchased from Miltenyi Biotec (Bergisch Gladbach, Germany), cell surface molecules were examined using anti-mouse antibodies including APCconjugated CD80, PE-conjugated CD86 (Miltenyi Biotec), and PE-conjugated, CD45, PerCP/ Cy5.5-conjugated CD11b, PE-conjugated CD11b, PE/ Cy7-conjugated Ly6G, Pacific Blue-conjugated Ly6C, FITC-conjugated F4/80, Brilliant Violet 510-conjugated MHC II, FITC-conjugated IFN- $\gamma$ (BioLegend, San Diego, CA, USA), and $\mathrm{T}$ cell proliferation was evaluated using a CellTrace ${ }^{\mathrm{TM}}$ CFSE Cell Proliferation Kit (Invitrogen, Thermo Fisher Scientific).

\section{Statistical Analysis}

Statistical analyses were performed using GraphPad Prism version 7 (San Diego, CA, USA). Data were presented as mean values $\pm \mathrm{SD}$. Student's $t$ test (paired, two-tailed, between 2 groups) or one-way analysis of variance (ANOVA, $\geq 3$ groups) was used to perform comparisons. $* \mathrm{P}<0.05$ was accepted as significantly different.

\section{Results}

\section{KAR Alleviates Visceral Hypersensitivity and Mucosal Inflammatory Responses in Mice with IBS}

To investigate whether KAR was effective for IBS, we established a TNBS-induced IBS mouse model. As shown in Figure 1A, IBS mice suffered from significantly increased visceral hypersensitivity compared with TNBSunexposed control mice. Next, we found that KAR treatment potently reduced the AWR scores at all distention volumes in a dose-dependent manner (Figure 1A, Supplementary Figure 1). This effect was also verified by visceromotor response (VMR) assessment (Figure 1B). In addition, the colon transportation test (CTT) was used to evaluate the intestinal motility alteration. We used the Bristol stool grade and monitored the first black stool time to show that the intestinal motility was obviously increased in IBS mice compared with TNBS-unexposed control mice, which was largely reversed by KAR treatment (Figure $1 \mathrm{C}$ and $\mathrm{D}$ ). These data suggest that KAR could effectively relief the clinical presentations in IBS mice. Furthermore, compromised intestinal barrier function and overexpression of inflammatory mediators have been indicated in the development of IBS. To this end, we first measured the permeability of the intestinal barrier by analyzing the FITC-dextran and LPS concentrations in the serum of mice, and found that serum FITC-dextran and LPS levels were greatly elevated in IBS mice, both of which were reduced by KAR treatment (Figure $1 \mathrm{E}$ and F). Subsequently, we examined levels of several inflammatory cytokines in the colon, and showed that KAR remarkably decreased MPO expression (an indicator of neutrophil activity, Figure $1 \mathrm{G}$ ) and suppressed the expression of IL-6, TNF- $\alpha$, and IL-1 $\beta$ in IBS mice. Notably, we found that colonic IL-10, a potent anti-inflammatory cytokine, was significantly up-regulated in KAR-treated IBS mice (Figure $1 \mathrm{H}$ ). These data suggest that KAR might exert a therapeutic effect on IBS via inhibiting mucosal inflammation and enhancing anti-inflammatory responses.

\section{KAR Mediates Macrophage Phenotype in the Colon of Mice with IBS}

Since macrophages were well-known to play a role in mucosal inflammation in intestinal inflammatory diseases. We analyzed the phenotype and function of colonic macrophages in IBS mice. We used an established flow cytometric gating strategy ${ }^{21,22}$ to determine macrophages (Figure 2A) and found that KAR treatment reduced the frequency of total macrophages $\left(\mathrm{CD} 45^{+} \mathrm{CD} 11 \mathrm{~b}^{+} \mathrm{CD} 11 \mathrm{c}^{-}\right.$ $\mathrm{Ly}_{6} \mathrm{G}^{-} \mathrm{F} 4 / 80^{+}$) in IBS colons (Figure 2B). Next, we analyzed the phenotype of macrophages by using additional markers (Ly6C and MHC II) and found that the frequency of pro-inflammatory macrophages $\left(\mathrm{CD}^{2} 5^{+} \mathrm{CD} 11 \mathrm{~b}^{+}\right.$ 


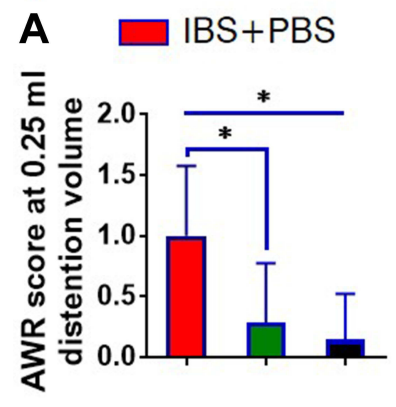

B

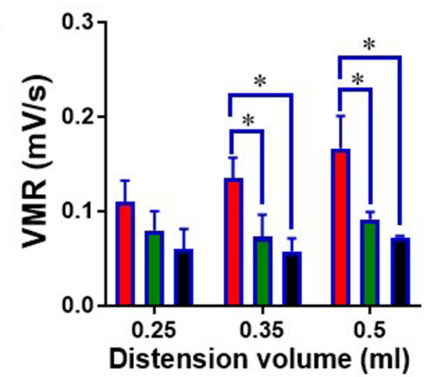

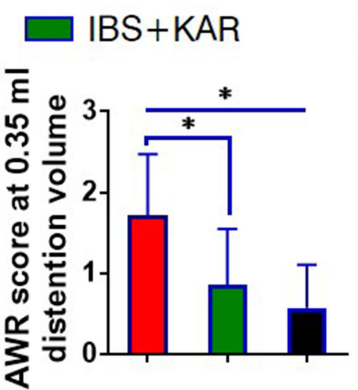

C

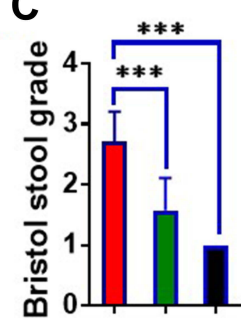

Controls

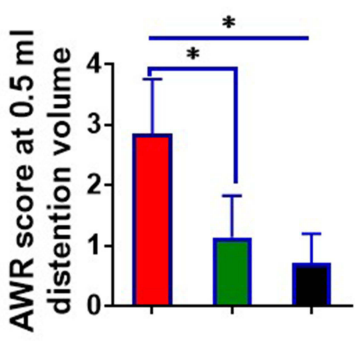

D

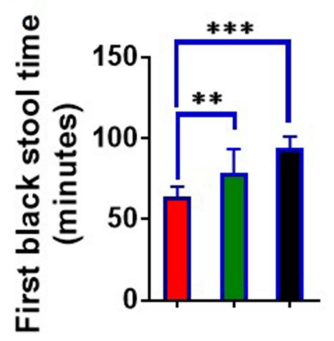

E

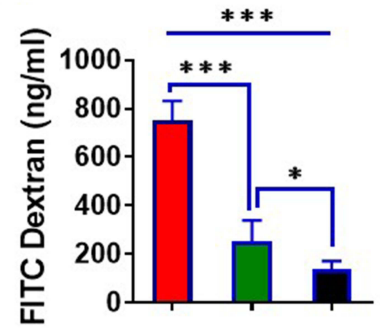

F

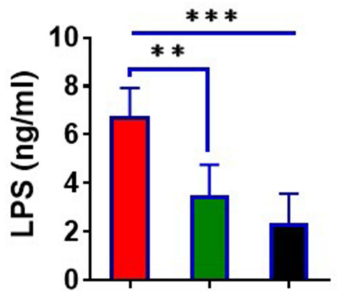

G

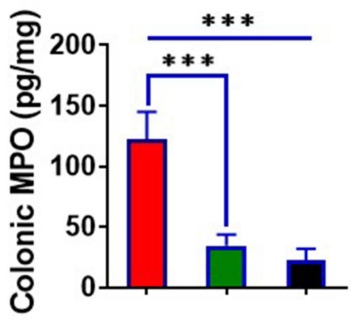

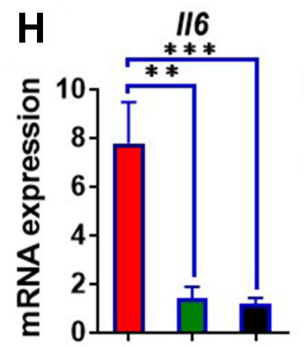
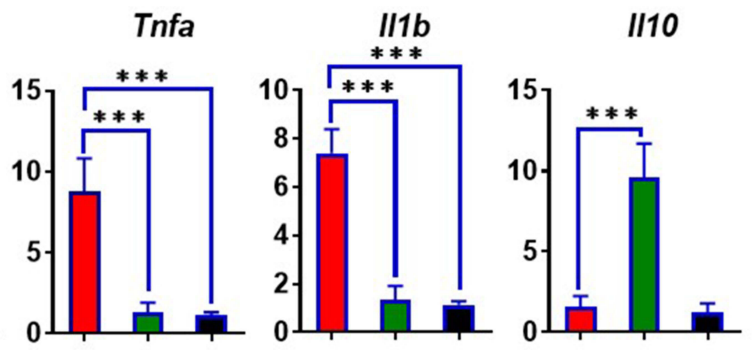

Figure I Kurarinone (KAR) alleviates visceral hypersensitivity and inflammatory responses in mice with irritable bowel syndrome (IBS). An IBS mouse model was established as indicated. IBS mice (wild type on a C57BL/B6] background) were daily received intraperitoneally with PBS or KAR (> 99\% purity, WuXi PharmaTech, Shanghai, China) at the dosage of $100 \mathrm{mg} / \mathrm{kg} /$ day beginning from 10 days post TNBS insults to the end of the experiment. Age- and gender-matched wild type (WT) mice that were not exposed to 2,4,6-trinitrobenzenesulfonic acid (TNBS) served as the control group. (A) The abdominal withdrawal reflex (AWR) test was performed to evaluate the visceral hypersensitivity in response to colorectal distention (CRD) in mice at distending volume of 0.25 , 0.35 , or $0.5 \mathrm{~mL}$. (B) Kinetic visceromotor response (VMR) to CRD at distending volume of $0.25,0.35$, or $0.5 \mathrm{~mL}$. (C) Bristol stool grade. (D) The first black stool time. Intestinal permeability was mirrored by serum (E) FITC-dextran and (F) LPS concentrations. (G) The level of MPO in the colon was measured to represent the activity of neutrophils. (H) The transcript expression levels of colonic IL-6, TNF- $\alpha$, IL$\mathrm{I} \beta$, and IL- I 0 were measured by $\mathrm{qRT}$-PCR. $* \mathrm{P}<0.05$, $* * \mathrm{P}<0.0 \mathrm{I}$, *** $\mathrm{P}<0.00 \mathrm{I}$, one-way analysis of variance (ANOVA). $\mathrm{n}=8$ mice in each group. Representative results from one of three independent experiments were shown.

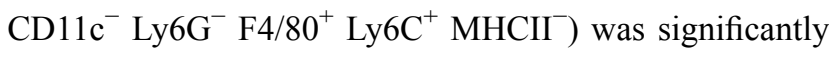
decreased (Figure $2 \mathrm{C}$ ) but the anti-inflammatory subset $\left(\mathrm{CD}^{+} 5^{+} \mathrm{CD}_{11 \mathrm{~b}^{+} \text {CD11 }}^{-} \mathrm{Ly}^{-} \mathrm{G}^{-} \mathrm{F} 4 / 80^{+} \mathrm{Ly}^{-} \mathrm{C}^{-}\right)$was upregulated (Figure 2D) in KAR-treated IBS mice than PBStreated IBS mice. Moreover, we sorted colonic macrophages from IBS mice with or without KAR by flow cytometry and measured a panel of cytokines and transcriptional factors. qRT-PCR revealed that KAR decreased colonic macrophage expression of IL- $1 \beta, \mathrm{TNF}-\alpha$ and iNOS, while increased their expression of Arg1 and IL10 (Figure 2E), suggesting that KAR might be able to inhibit pro-inflammatory responses in macrophages by imprinting an immunoregulatory property on them.

\section{KAR Mediates Macrophage Phenotype and Function via AhR}

We next sought to further investigate whether KAR could indeed modulate macrophage function and explore the underlying mechanisms. To this end, we generated BMDM and stimulated in vitro them with LPS in the presence or absence of KAR to evaluate its effect on macrophages. KAR markedly down-regulated LPSinduced macrophage activation, characterized by lower levels of CD86 and CD80 than controls (Figure 3A and B). We also analyzed the expression of pro- and antiinflammatory genes, and found that LPS-stimulated macrophages with KAR were less pro-inflammatory in 

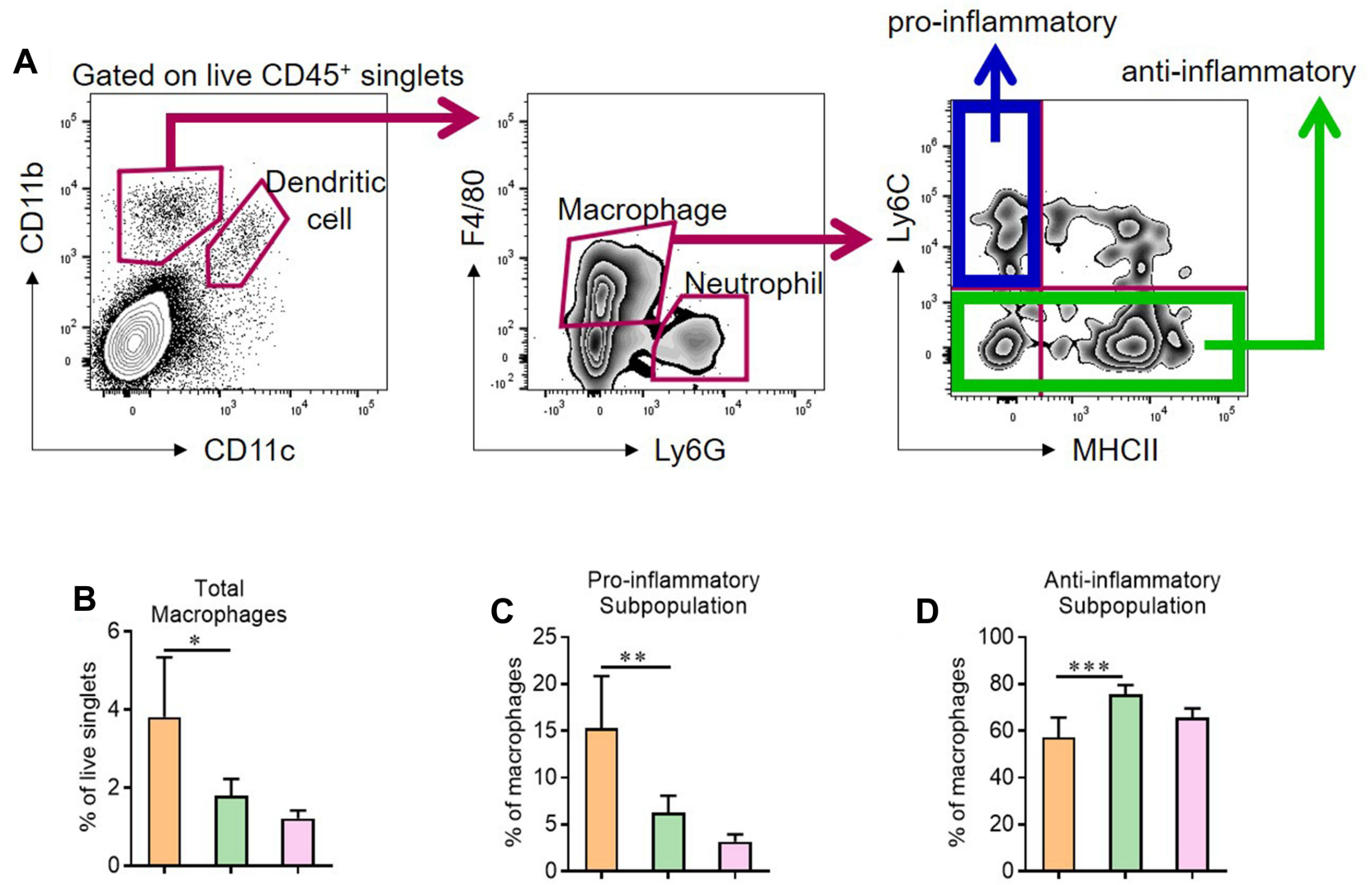

E
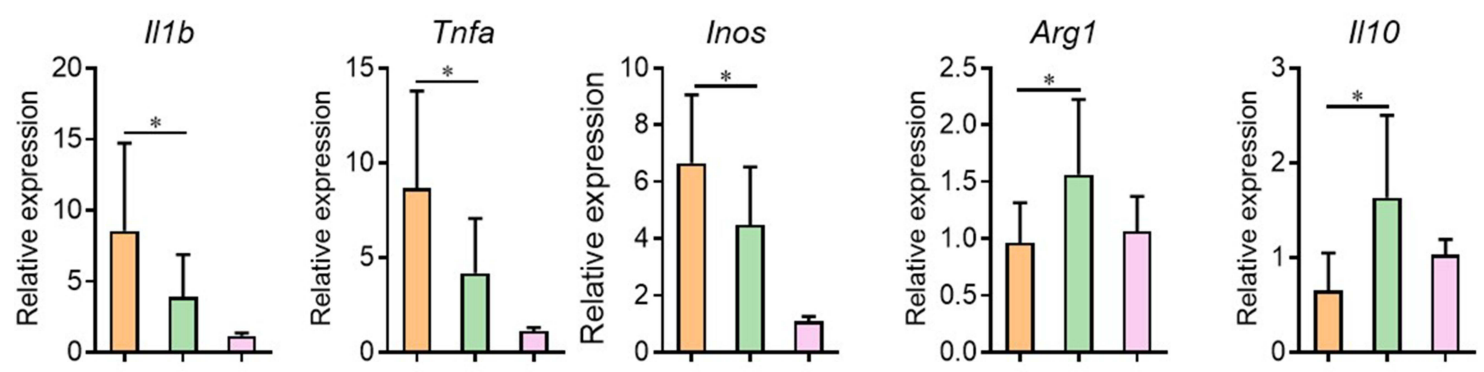

Figure 2 KAR mediates macrophage phenotype in the colon of mice with IBS. IBS mouse model was established and KAR was administrated as described in Figure I.

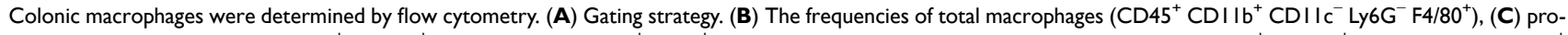

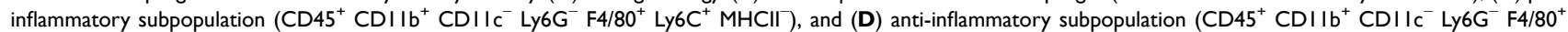
Ly $6 \mathrm{C}^{-}$) were presented in bar charts. (E) Colonic macrophages were sorted by flow cytometry and their transcript expression levels of IL-I $\beta$, TNF- $\alpha$, iNOS, ArgI and IL-I0 were measured by qRT-PCR. $* P<0.05, * * P<0.01$, $* * * P<0.001$, one-way analysis of variance (ANOVA). $n=8$ mice in each group. Representative results from one of three independent experiments were shown.

nature as evidenced by lower expression of inflammatory genes (Illb, Tnfa, Il12) (Figure 3C) but higher expression of anti-inflammatory genes (Argl, Fizzl, Ill0) (Figure 3D). As presented in Supplementary Figure 2, the effect of KAR on LPS-stimulated BMDM was dosedependent. Given macrophages have capacities to affect $\mathrm{T}$ cell activation and proliferation, we next co-cultured KAR-treated or non-treated BMDM with naïve $\mathrm{CD} 4^{+}$
T cells and used a CFSE Cell Proliferation Kit to determine $\mathrm{T}$ cell proliferation. KAR-treated macrophages greatly inhibited $\mathrm{T}$ cell proliferation (Figure 3E). Flow cytometric staining for $\mathrm{CD} 62 \mathrm{~L}$, a naïve $\mathrm{T}$ cell marker, showed that $\mathrm{T}$ cell activation was suppressed by KARtreated macrophages either (Figure 3F). These data are indicative of an immunoregulatory phenotype of KARtreated macrophages. 
A

A
ป⿱
0
0
$\overline{0}$
0

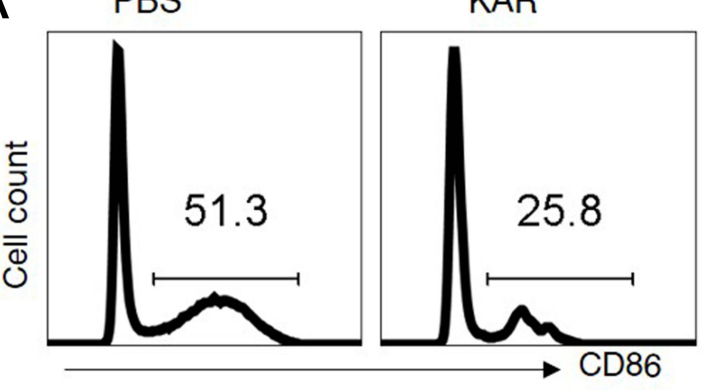

B

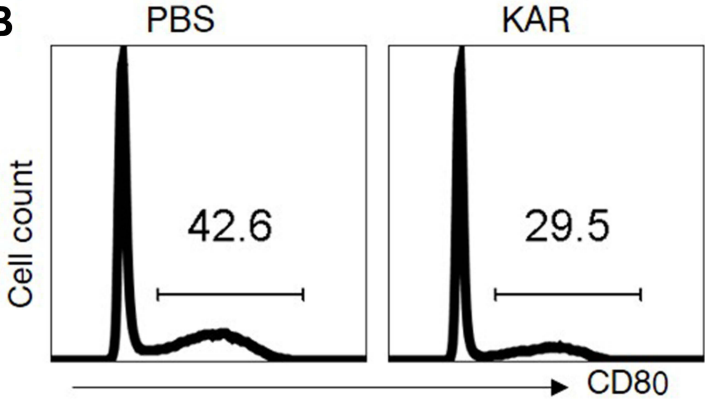

E

T cells cultured with: PBS-BMDM

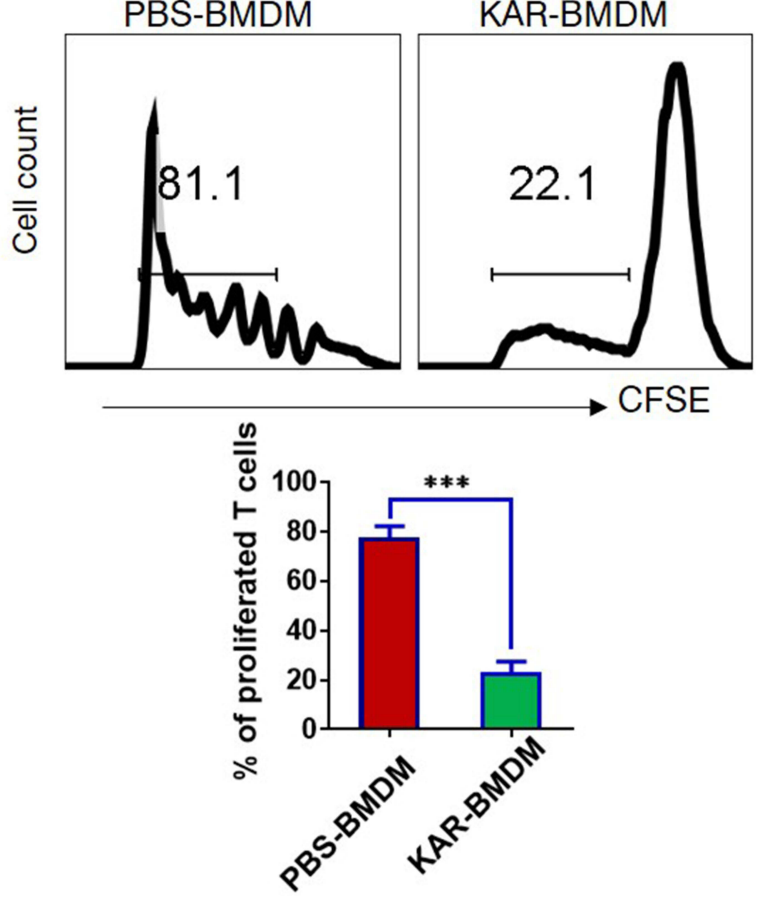

C

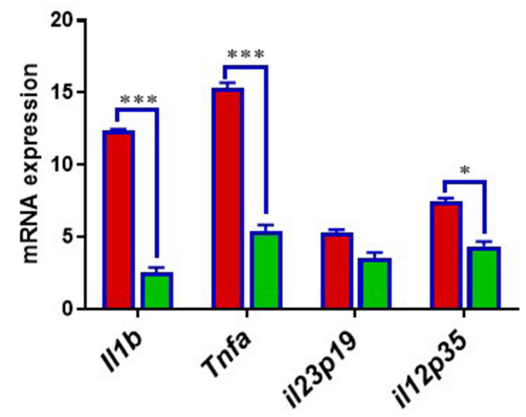

D

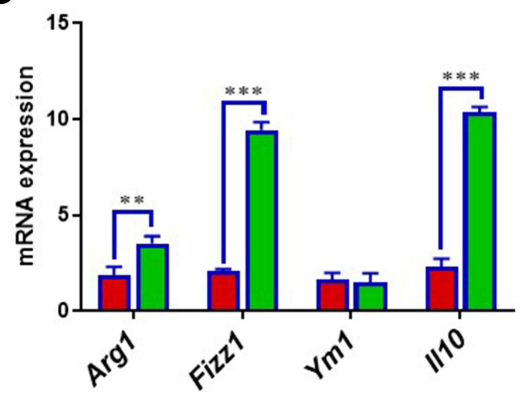

F

T cells cultured with:
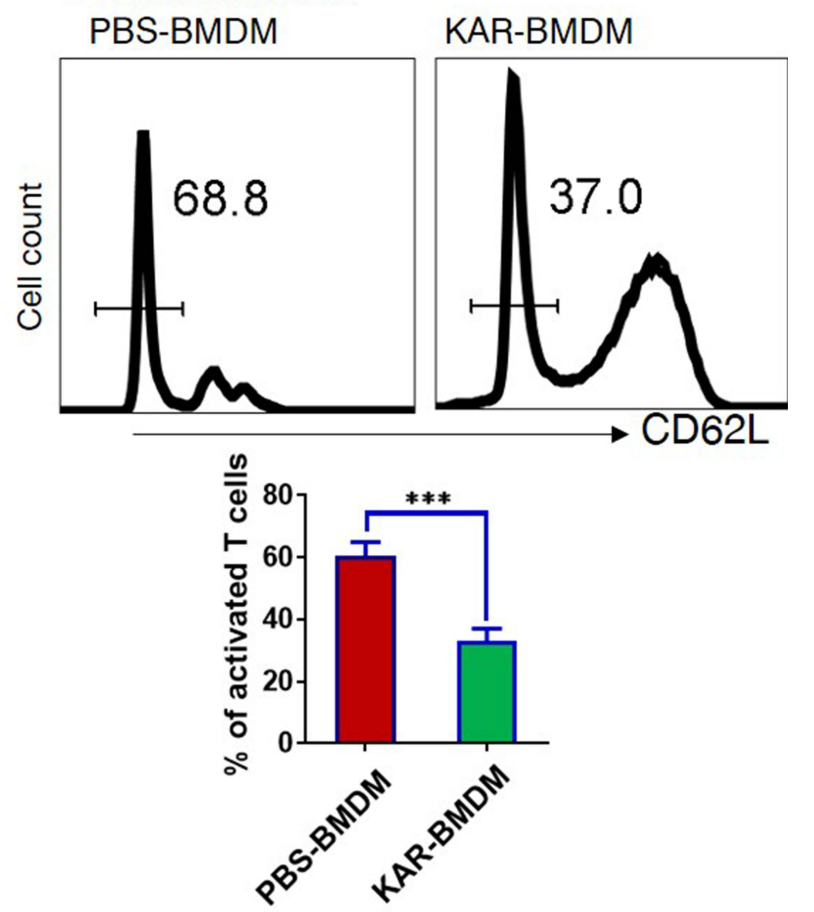

Figure 3 KAR mediates macrophage phenotype and function. (A) Mouse bone marrow-derived macrophages (BMDM) were stimulated with LPS (I00 ng/mL) and incubated in vitro with $\mathrm{KAR}(20 \mu \mathrm{g} / \mathrm{mL})$ or phosphate buffer saline (PBS). After 2 days of stimulation, flow cytometry was performed to analyze (A) CD86 and (B) CD80 expression on BMDC. (C and D) qRT-PCR was performed to determine the expression levels of indicated genes. Two groups of BMDM were obtained as described above and co-cultured with splenic naïve $\mathrm{CD}^{+} \mathrm{T}$ cells pre-labelled with carboxyfluorescein succinimidyl ester (CFSE) in the presence of anti-CD3/CD28. The ratio of T cells: BMDM was 4:I. After 5 days of co-culture, flow cytometry was performed to analyze $(\mathbf{E})$ the dilution of CFSE intensity and (F) the surface expression level of CD62L. $* \mathrm{P}<0.05$, $* * \mathrm{P}<0.0 \mathrm{I}$, $* * * \mathrm{P}<$ 0.00 I, Student's $t$ test. Triplicates were carried out in each experiment. Representative results from one of three independent experiments were shown. 
Next, we further investigated the downstream pathways in the KAR regulation of macrophage functions. Previous studies have suggested a potential role of AhR in shifting macrophages toward an anti-inflammatory phenotype, ${ }^{23}$ we asked whether AhR was involved in the effect of KAR on macrophages. Towards this direction, we first verified that KAR treatment indeed up-regulated AhR expression in BMDM (Figure 4A). We found that LPS induced a pro-inflammatory nature in $\mathrm{AhR}^{-/-} \mathrm{BMDM}$ to a larger extent than $\mathrm{AhR}^{+/+} \mathrm{BMDM}$ (Figure 4B). Additionally, AhR deficiency greatly impaired the inhibitory effect of KAR on BMDM inflammatory cytokine expression under LPS stimulation, compared with $\mathrm{AhR}^{+/+}$ BMDM (Figure 4B). Importantly, AhR deficiency abrogated KAR-induced anti-inflammatory mediator expression in BMDM (Figure 4C). Expectedly, AhR deficiency impaired the ability of KAR-treated BMDM to inhibit $\mathrm{T}$ cell proliferation (Figure 4D) and IFN- $\gamma$ expression (Figure 4E). Taken together, KAR might imprint a regulatory phenotype on macrophages via AhR.

\section{AhR Deficiency in Macrophages Impaired the Effect of KAR on IBS Mice}

Next, we wanted to investigate the role of macrophagederived AhR in the effect of KAR on IBS. First of all, we found that $\mathrm{AhR}^{-/-}$mice were more susceptible to TNBSinduced IBS than $\mathrm{AhR}^{+/+}$littermates (Supplementary Figure 3) and AhR expression in colonic macrophages was up-regulated in IBS mice with KAR compared to those without KAR (Supplementary Figure 4), prompting us to believe that macrophage-derived AhR might be crucial for intestinal homeostasis and involved in the effect of KAR on IBS.

Next, we administrated CD11b-DTR transgenic mice with TNBS to establish the IBS model and macrophages were depleted in these mice using diphtheria toxin (DTx) 7 days after TNBS enema. As shown in Supplementary Figure 5, there were no evident differences in the susceptibility to TNBS between CD11b-DTR and their wild type (WT) littermates. In addition, we observed that CD11bDTR mice with macrophage depletion suffered from milder visceral hypersensitivity (Supplementary Figure 6), and transfer of macrophages restored the visceral hypersensitivity to a level comparable to CD11b-DTR mice without DTx (Supplementary Figure 6). Subsequently, peritoneal macrophages isolated from $\mathrm{AhR}^{+/+}$or $\mathrm{AhR}^{-/-}$mice were transferred into DTx-treated
CD11b-DTR transgenic mice to reconstitute them (Figure 5A). The visceral sense and intestinal motility were measured in all groups of mice. We found that IBS mice with $\mathrm{AhR}^{-/-}$macrophages exhibited increased visceral sense and intestinal motility compared to those with $\mathrm{AhR}^{+/+}$macrophages, and the therapeutic effect of KAR on IBS was significantly impaired in TNBS-exposed chimeras with $\mathrm{AhR}^{-/}$macrophages compared to that in their counterparts with $\mathrm{AhR}^{+/+}$macrophages (Figure 5B-E). Regarding intestinal barrier permeability, KAR-treated IBS chimeras with $\mathrm{AhR}^{-/-}$macrophages exhibited much higher FITC Dextran concentration in the serum compared to KAR-treated IBS chimeras with $\mathrm{AhR}^{+/+}$macrophages (Figure 5F). Additionally, we investigated whether AhR deficiency in macrophages could abrogate the suppressive effect of KAR on mucosal inflammation in IBS mice. Colonic inflammatory gene expression including $I l l b$, Il12, Tnfa and Inos was potently reduced and antiinflammatory gene expression including Argl, Fizzl, $Y m 1$, and $\mathrm{Il}-10$ was increased by KAR in IBS chimeras with $\mathrm{AhR}^{+/+}$macrophages compared to non-treated IBS mice, but AhR deficiency in macrophages significantly abrogated these effects (Figure 5G and H). Collectively, these findings suggest that KAR could ameliorate IBS in mice and macrophage-derived AhR plays a crucial role in this effect.

\section{KAR Regulates the Functions of Human Monocyte-Derived Macrophages (MDM) via $A h R$, Which is Down-Regulated in Patients with IBS}

We next sought to validate our findings above in human macrophages. We first generated human MDM and confirmed that KAR indeed up-regulated AhR expression in MDM (Figure 6A). Next, we treated MDM with or without KAR under stimulation of LPS. Similar with observations in mice, KAR significantly decreased LPS-induced $T N F a$ and $I l l b$ gene expression but increased $I l 10$ gene expression in MDM, and knockdown of AhR significantly abrogated these effects (Figure 6B). Subsequently, we analyzed AhR expression profile in patients with IBS. We found that AhR expression in either mucosal tissues (Figure 6C) or MDM (Figure 6D) was significantly decreased in all three IBS groups (IBS-C, IBS-D, and IBS-M) compared to that in healthy control group. Furthermore, we revealed that AhR expression in MDM from IBS patients was positively correlated with serum IL- 
A

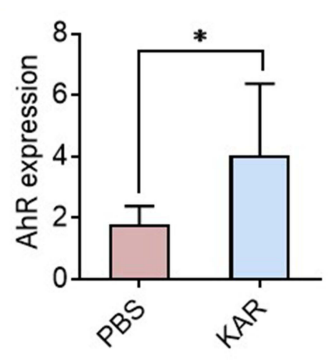

D

T cells cultured with:
B
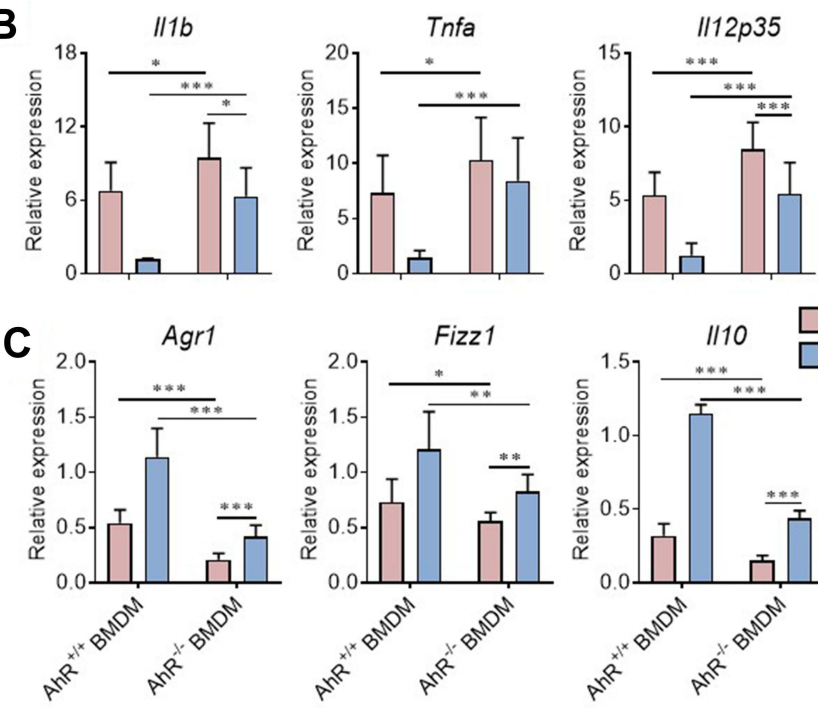
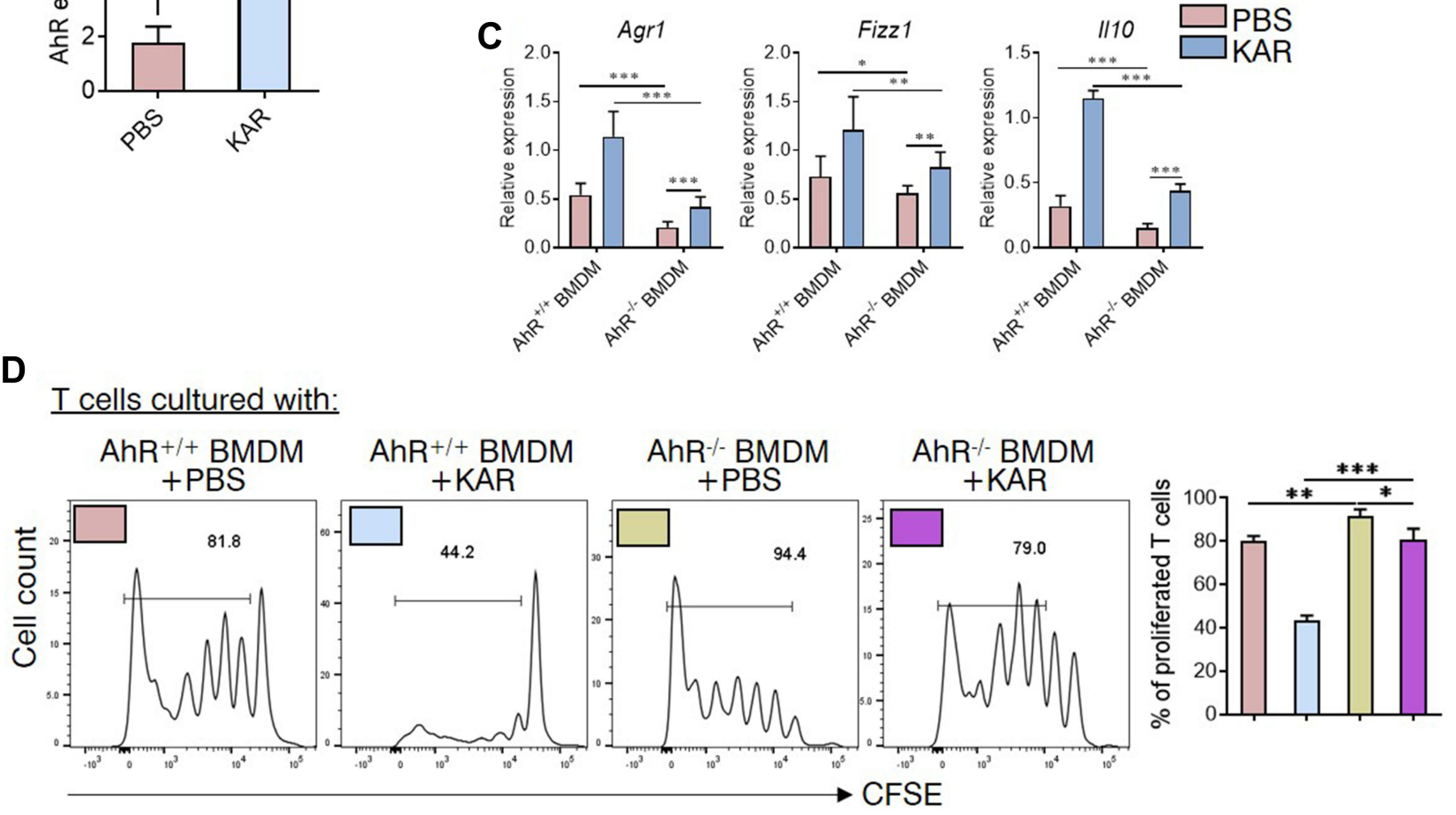

E

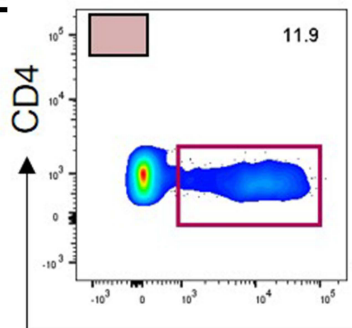

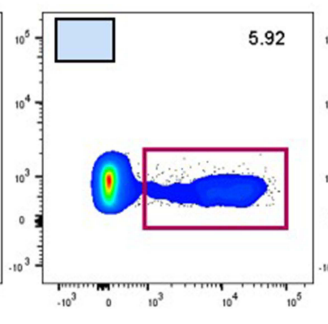

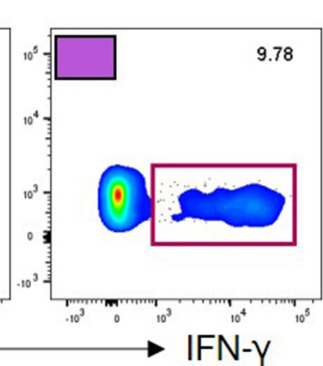

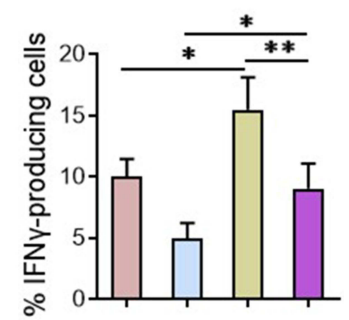

Figure 4 KAR mediates macrophage phenotype and function via aryl hydrocarbon receptor (AhR). (A) Mouse bone marrow-derived macrophages (BMDM) were generated as indicated and incubated in vitro with KAR $(20 \mu \mathrm{g} / \mathrm{mL})$ or PBS. AhR mRNA expression was examined using qRT-PCR. *P < 0.05 , Student's $t$ test. (B-D) BMDM were generated from $\mathrm{AhR}^{+/+}$or $\mathrm{AhR}^{-/-}$mice. Four groups of BMDM cultures were set up: LPS ( $100 \mathrm{ng} / \mathrm{mL}$, Sigma-Aldrich, St. Louis, MO, USA)-activated AhR ${ }^{+/+}$BMDM with PBS, LPS-activated $\mathrm{AhR}^{+/+}$BMDM with KAR, LPS-activated AhR ${ }^{-/-}$BMDM with PBS and LPS-activated AhR ${ }^{-/-}$BMDM with KAR. After 2 days of culture, BMDM were harvested and qRT-PCR was performed to determine the expression levels of indicated genes (B and $\mathbf{C}$ ). Four groups of BMDM were obtained as described in (B and $\mathbf{C})$ and cocultured with splenic naïv $\mathrm{CD} 4^{+} \mathrm{T}$ cells pre-labelled with carboxyfluorescein succinimidyl ester (CFSE) in the presence of anti-CD3/CD28. The ratio of T cells: BMDM was 4:I. After 5 days of co-culture, flow cytometry was performed to analyze (D) the dilution of CFSE intensity and (E) the expression of IFN- $\gamma$. (B-E) $*$ P $<0.05$, **P < 0.0 I, *** $\mathrm{P}<0.00 \mathrm{I}$, one-way analysis of variance (ANOVA). Triplicates were carried out in each experiment. Representative results from one of three independent experiments were shown.

10 protein level (Figure 6E) and yet inversely correlated with serum TNF- $\alpha$ protein level (Figure 6F). As shown in Figure 6G, we used serum LPS concentration to mirror the intestinal barrier permeability in IBS patients, and found that AhR expression in MDM was negatively correlated with serum LPS level. It has been well-known that IBS patients often suffer from anxiety and depression during the course of the disease. ${ }^{24}$ We found that the levels of AhR in MDM were negatively correlated with anxiety scores (HAD-A) in patients with IBS (Figure 6H). 

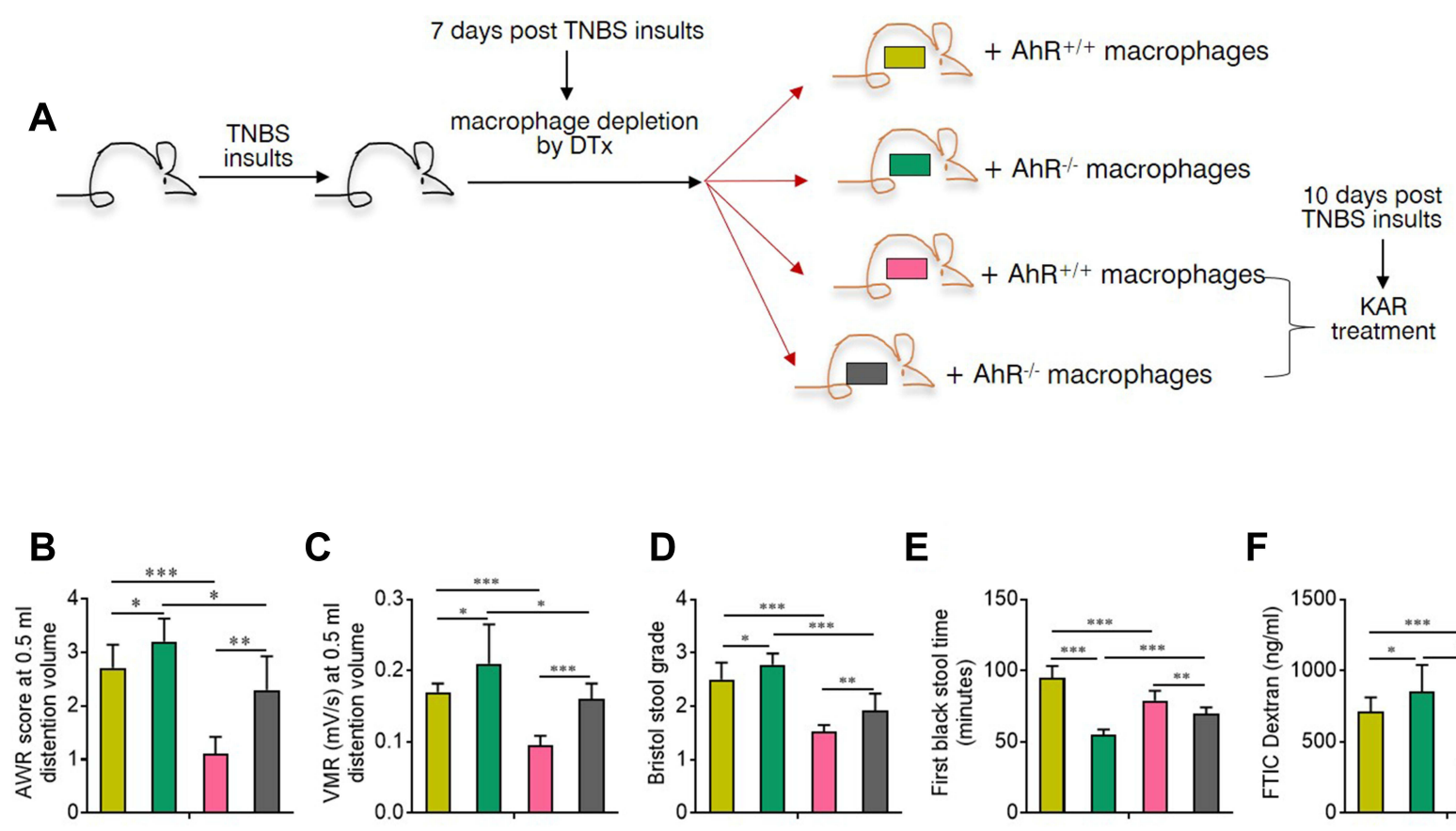

E

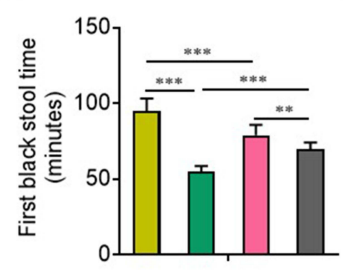

F

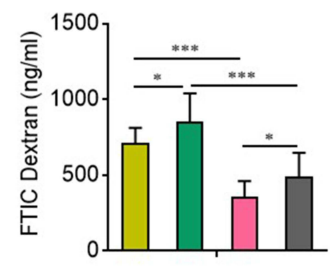

G

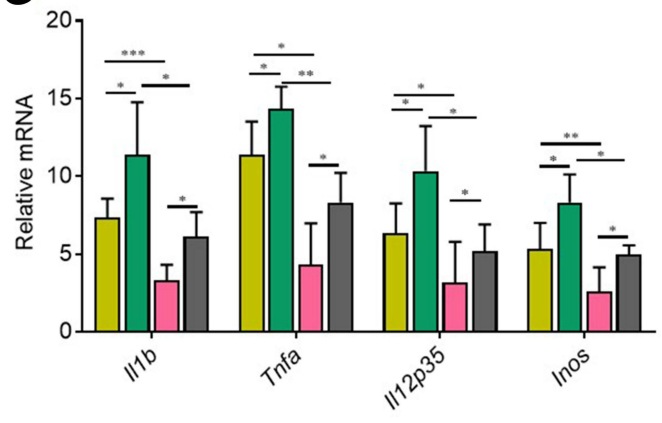

H

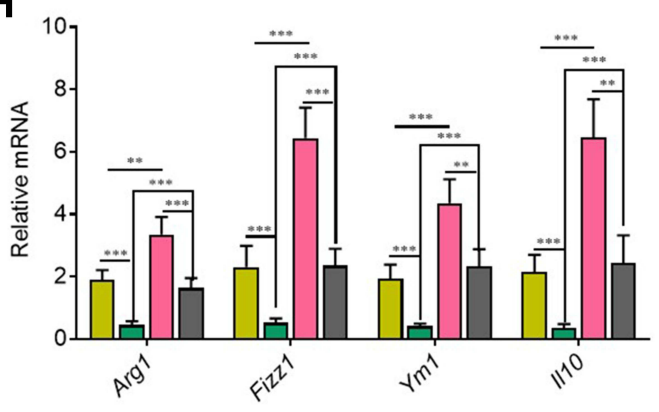

Figure 5 AhR deficiency in macrophages impaired the effect of KAR on IBS mice. (A) CDII lb-DTR transgenic mice were received with TNBS to induce colonic inflammation as in Figure I, and DTx $(2 \mathrm{mg} / \mathrm{g})$ was injected intraperitoneally into them to deplete macrophages 7 days post TNBS exposure. Peritoneal macrophages from $\mathrm{AhR}^{+/+}$or $\mathrm{AhR}^{-/-}$mice were purified and were transferred intravenously into DTx-treated mice. $2 \times 10^{6}$ macrophages for each DTx-treated mouse. After 3 days of reconstitution, KAR $\left(100 \mathrm{mg} / \mathrm{kg} /\right.$ day) was administrated intraperitoneally and these chimeras were divided into four groups $(\mathrm{n}=8)$ : chimeras with $A h R^{+/+}$macrophages $\left(\mathrm{AhR}^{+/+}\right.$chimeras) without KAR, $\mathrm{AhR}^{+/+}$chimeras with $\mathrm{KAR}$ treatment, chimeras with $\mathrm{AhR}^{-/-}$macrophages (AhR ${ }^{-/-}$chimeras) without $\mathrm{KAR}$, and $\mathrm{AhR} \mathrm{R}^{-/-}$chimeras with $\mathrm{KAR}$ treatment. The (B) AWR test and (C) VMR in response to CRD at $0.5 \mathrm{~mL}$ distending volume. (D) Bristol stool grade. (E) The first black stool time. (F) Intestinal permeability was minored by serum FITC-dextran concentrations. The transcript expression levels of colonic (D) inflammatory mediators (IL-I $\beta$, TNF- $\alpha$, II-I2p35, and iNOS) and anti-inflammatory mediators (Argl, Fizzl, YmI, and IL-I0) were measured by qRT-PCR. *P $<0.05$, **P $<0.0$, *** $2<0.00 \mathrm{I}$, one-way analysis of variance (ANOVA). Representative results from one of three independent experiments were shown.

\section{Discussion}

A great number of studies have provided compelling evidences that macrophages are critical players as a member of innate immunity and a trainer of adaptive immune responses in the development of IBS, suggesting that targeting macrophages could assist to develop potential drugs for IBS. Our current study demonstrated a therapeutic role of KAR on IBS in mice via regulating macrophage functions through activating AhR signaling.
So far, many medicines currently used in clinic have various bioactive compounds derived from natural plants. Flavonoids widely exist in natural plants, which are a group of naturally-occurring botanical compounds derived from 2-phenylchromone. ${ }^{25}$ KAR, a member of the flavonoid family is derived from Sophora flavescens, has been found to exert potent therapeutic effects in many disorders, including inflammation and cancers. KAR was able to inhibit the growth and induce the mitochondria 
A

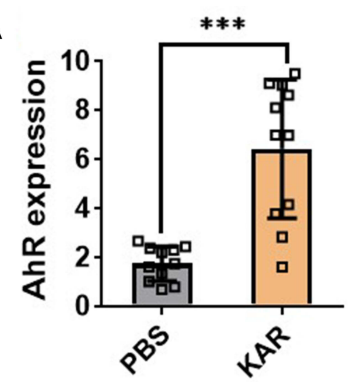

C

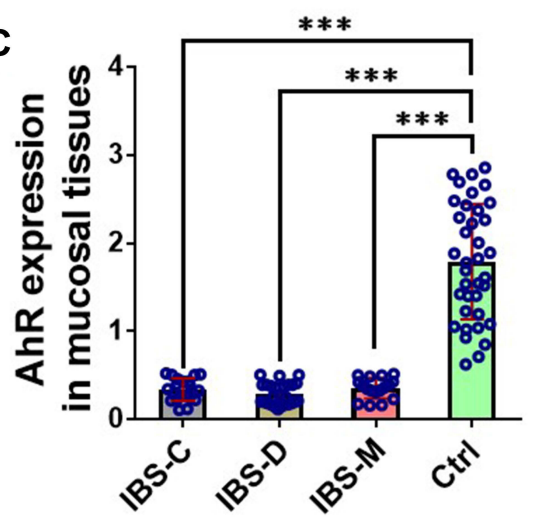

E

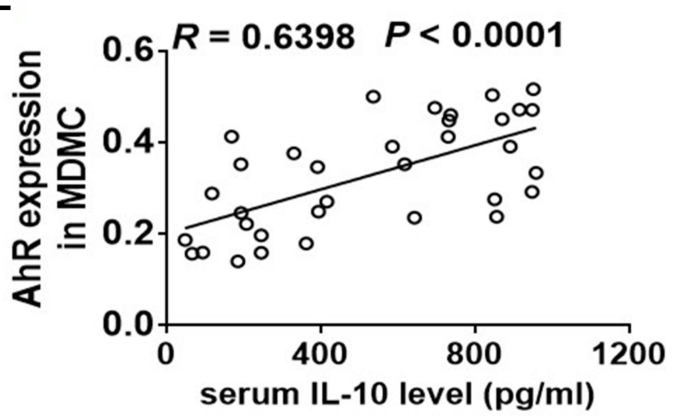

G

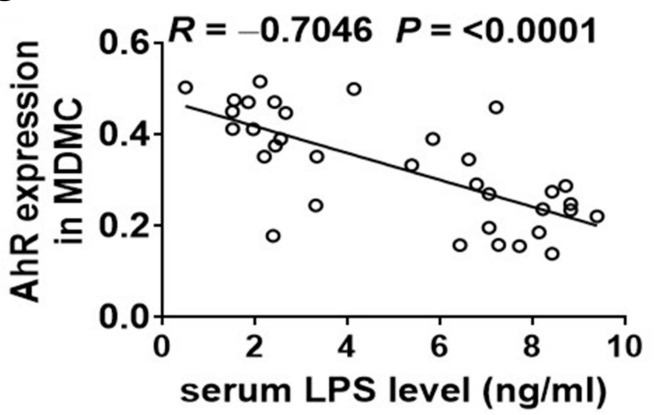

Ctrl siRNA-MDM+KAR

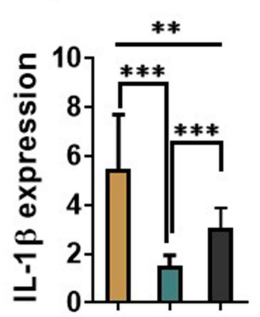

AhR siRNA-MDM+KAR
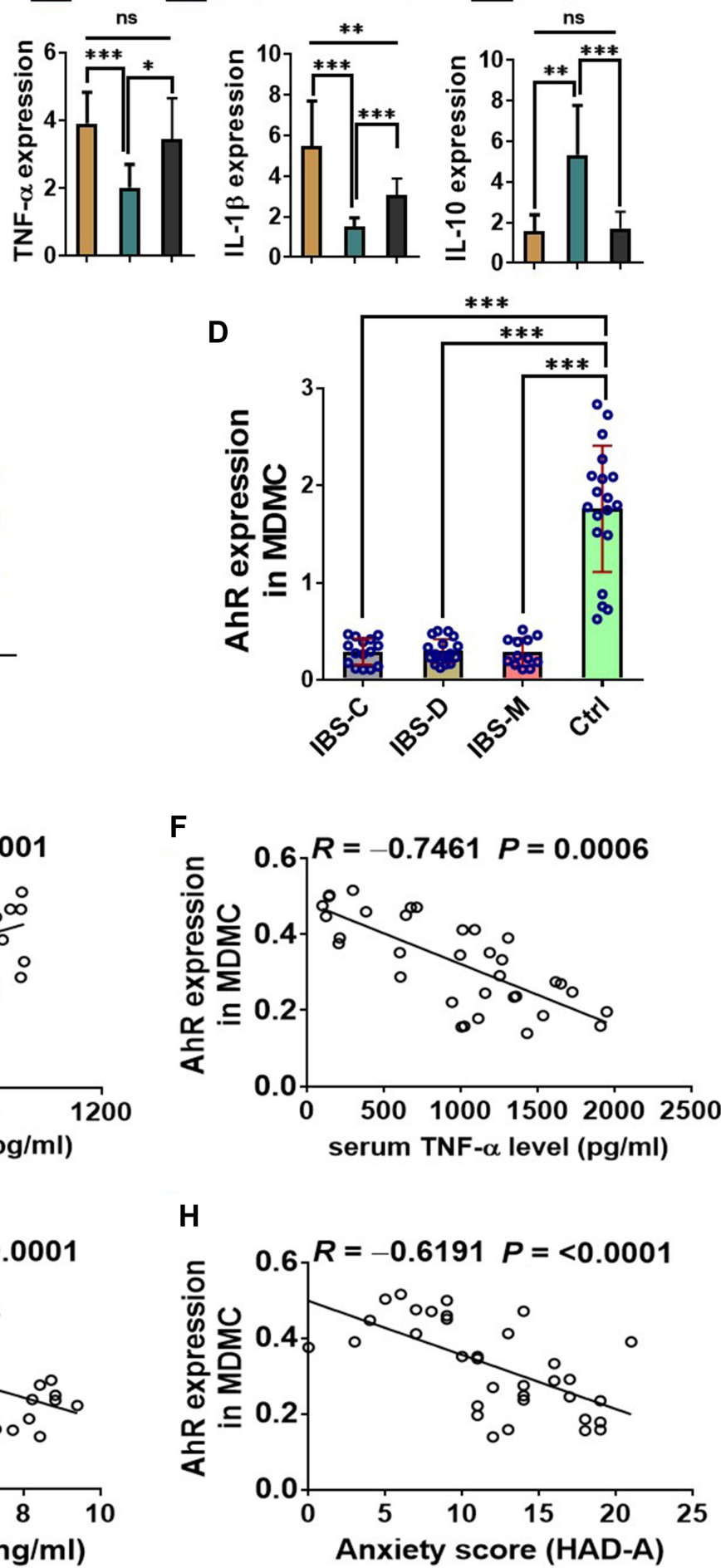

Figure 6 KAR regulates the functions of human monocyte-derived macrophages (MDM) via AhR, which is down-regulated in patients with IBS. (A) Human MDM were generated from peripheral blood monocytes of healthy donors $(n=I I)$. MDM were incubated in vitro with or without $\mathrm{KAR}(20 \mu \mathrm{g} / \mathrm{mL})$ for 48 hours. AhR expression in MDM was examined by qRT-PCR. ***P < $0.00 \mathrm{I}$, Student's $t$ test. Representative results from one of three independent experiments were shown. (B) MDM were transfected with or without AhR siRNA, and stimulated with LPS in the presence of KAR. LPS-stimulated non-transfected MDM without KAR treatment served as controls. After 2 days of incubation, MDM were harvested and qRT-PCR was performed to determine the expression levels of cytokines. (C) Colonic biopsies from patients with constipationpredominant IBS (IBS-C, $n=21$ ), diarrhea-predominant IBS (IBS-D, $n=26)$, IBS with mixed bowel habits (IBS-M, $n=17)$, and healthy controls (Ctrl, $n=37)$ were collected and their expression of AhR was examined by qRT-PCR. (D) Peripheral blood monocytes were obtained from patients with IBS-C (I5/2I), IBS-D (I8/26), IBS-M (I2/I7), and healthy controls (19/37) described in (C), and MDM were generated in vitro. AhR was examined by qRT-PCR. (B-D) $* P<0.05$, **P $<0.0 \mathrm{I}$, $* * * P<0.00 \mathrm{I}$, one-way analysis of variance (ANOVA). Serum IL-10, TNF- $\alpha$, and LPS concentrations were measured in 34 IBS patients. Correlation analysis between IBS MDM expression of AhR and serum (E) IL-10, (F) TNF- $\alpha$, and (G) LPS concentrations, respectively. (H) Correlation between MDM expression of AhR and anxiety scores according to Hospital Anxiety and Depression Scale (HAD-A) in IBS patients $(n=34)$. $(\mathbf{E}-\mathbf{H})$ Pearson's correlation.

Abbreviation: ns, no statistical significance. 
apoptosis of non-small cell lung cancer (NSCLC) cells both in vivo and in vitro without noticeable toxic effects. $^{11}$ In EAE, a mouse model of human multiple sclerosis, KAR greatly relieved clinical symptoms and suppressed $\mathrm{T}$ cell-mediated autoimmune responses in the CNS of EAE mice, suggesting an immunomodulatory role of KAR. ${ }^{12}$ Moreover, KAR could abrogate $\mathrm{CD}^{+} \mathrm{T}$ cell differentiation via inhibiting JAK/STAT signaling and TCR activation and thus repressed the development of two inflammatory skin diseases. ${ }^{26}$ Previous data demonstrated that KAR was able to suppress macrophage production of inflammatory mediators upon LPS stimulation, ${ }^{14}$ we further investigated this effect in IBS and the underlying mechanisms. We found that KAR markedly inhibited LPS-induced BMDM activation, inflammatory gene expression, and their capacities of assisting $\mathrm{T}$ cell proliferation. More than that, KAR was found to up-regulate IL-10 expression in BMDM in vitro and in the colon of IBS mice, suggesting that KAR might imprint macrophage an immunoregulatory nature.

Additionally, we found that AhR was functionally involved in the effect of KAR on macrophage functions. AhR is widely expressed among immune, epithelial, and endothelial cells, and a member of the basic helix-loophelix transcription factor family. ${ }^{27} \mathrm{AhR}$ is able to bind with a panel of exogenous ligands including natural plant flavonoids, and regulate the maturation and function of many important immune cells in a ligand-dependent manner at multiple levels, including T cells, dendritic cells, and macrophages. $^{27}$ Recent evidences suggest that macrophages displayed rapid activation of $\mathrm{AhR}$ upon exposure to apoptotic cells, which led to massive IL-10 production. Myeloid cells deficient in AhR led to exaggerative systemic autoimmune responses in lupus prone mice. ${ }^{28}$ In the current study, we found that KAR significantly upregulated AhR expression in both mouse and human macrophages, and KAR failed to efficiently induce antiinflammatory gene expression including IL-10 by macrophages in the absence of AhR. Mice with specific deficiency of AhR in macrophages were more susceptible to IBS and the effect of KAR treatment on IBS was significantly impaired in these mice, suggesting a critical role of AhR in KAR regulation of macrophage functions and in the protection of IBS. Of note, the effect of KAR was not completely abrogated by AhR deficiency in IBS, indicating that there might be other downstream pathways involved in the pathogenesis of KAR in regulation of IBS.
In summary, we here revealed that KAR, a natural product derived from the roots of Sophora flavescens, acted as an effective drug for visceral hypersensitivity in IBS, and an essential regulator of mucosal inflammation through macrophage-intrinsic AhR signaling. Our findings provide new evidence that KAR might be a promising candidate as a therapeutic cure for IBS.

\section{Abbreviations}

IFN, interferon; IL, interleukin; TCR, T cell receptor; TNF, tumour necrosis factor; PBS, phosphate buffered saline; PCR, polymerase chain reaction; LPS, lipopolysaccharide; MPO, myeloperoxidase.

\section{Ethics Approval and Consent to Participate}

All procedures involving animals were approved and performed in accordance with the Animal Care and Use Committee at Sichuan Provincial People's Hospital. Human studies were conducted in accordance with the Declaration of Helsinki and approved by the Institutional Review Board for Clinical Research of the Sichuan Provincial People's Hospital.

\section{Funding}

This research was funded by Scientific Research Fund of Sichuan Provincial People's Hospital (No. 2016LY11).

\section{Disclosure}

The authors declare that they have no competing interests.

\section{References}

1. Black CJ, Ford AC. Global burden of irritable bowel syndrome: trends, predictions and risk factors. Nat Rev Gastroenterol Hepatol. 2020;17:473-486. doi:10.1038/s41575-020-0286-8

2. Ng QX, Soh AYS, Loke W, Lim DY, Yeo WS. The role of inflammation in irritable bowel syndrome (IBS. $J$ Inflamm Res. 2018;11:345-349. doi:10.2147/JIR.S174982

3. Liebregts T, Adam B, Bredack C, et al. Immune activation in patients with irritable bowel syndrome. Gastroenterology. 2007;132:913-920. doi:10.1053/j.gastro.2007.01.046

4. El-Salhy M, Gundersen D, Hatlebakk JG, Hausken T. Low-grade inflammation in the rectum of patients with sporadic irritable bowel syndrome. Mol Med Rep. 2013;7:1081-1085. doi:10.3892/ mmr.2013.1320

5. Chang L, Adeyemo M, Karagiannidis I, et al. Serum and colonic mucosal immune markers in irritable bowel syndrome. $\mathrm{Am}$ J Gastroenterol. 2012;107:262-272. doi:10.1038/ajg.2011.423

6. Barbara G, Stanghellini V, De Giorgio R, et al. Activated mast cells in proximity to colonic nerves correlate with abdominal pain in irritable bowel syndrome. Gastroenterology. 2004;126:693-702. doi:10.1053/j. gastro.2003.11.055 
7. Lee KJ, Kim YB, Kim JH, et al. The alteration of enterochromaffin cell, mast cell, and lamina propria $\mathrm{T}$ lymphocyte numbers in irritable bowel syndrome and its relationship with psychological factors. J Gastroenterol Hepatol. 2008;23:1689-1694. doi:10.1111/j.14401746.2008.05574.x

8. Park JH, Rhee P-L, Kim HS, et al. Mucosal mast cell counts correlate with visceral hypersensitivity in patients with diarrhea predominan irritable bowel syndrome. J Gastroenterol Hepatol. 2006;21:71-78. doi:10.1111/j.1440-1746.2005.04143.x

9. Ruder B, Becker C. At the Forefront of the Mucosal Barrier: the Role of Macrophages in the Intestine. Cells. 2020;9:2162. doi:10.3390/ cells9102162

10. Ford AC, Sperber AD, Corsetti M, Camilleri M. Irritable bowel syndrome. Lancet. 2020;396:1675-1688. doi:10.1016/S01406736(20)31548-8

11. Yang J, Chen H, Wang Q, et al. Inhibitory Effect of Kurarinone on Growth of Human Non-small Cell Lung Cancer: an Experimental Study Both in Vitro and in Vivo Studies. Front Pharmacol. 2018;9:252. doi:10.3389/fphar.2018.00252

12. Xie L, Gong W, Chen J, et al. The flavonoid kurarinone inhibits clinical progression of EAE through inhibiting Th1 and Th17 cell differentiation and proliferation. Int Immunopharmacol. 2018;62:227-236. doi:10.1016/j.intimp.2018.06.022

13. Chung TW, Lin CC, Lin SC, Chan HL, Yang CC. Antitumor effect of kurarinone and underlying mechanism in small cell lung carcinoma cells. Onco Targets Ther. 2019;12:6119-6131. doi:10.2147/OTT. S214964

14. Nishikawa S, Inoue Y, Hori Y, et al. Anti-Inflammatory Activity of Kurarinone Involves Induction of $\mathrm{HO}-1$ via the KEAP1/Nrf2 Pathway. Antioxidants (Basel. 2020;9:842.

15. Yi Q, Wang J, Song Y, et al. Ascl2 facilitates IL-10 production in Th17 cells to restrain their pathogenicity in inflammatory bowe disease. Biochem Biophys Res Commun. 2019;510(3):435-441. doi:10.1016/j.bbrc.2019.01.123

16. Xiu W, Chen Q, Wang Z, Wang J, Zhou Z. Microbiota-derived short chain fatty acid promotion of Amphiregulin expression by dendritic cells is regulated by GPR43 and Blimp-1. Biochem Biophys Res Commun. 2020;533:282-288. doi:10.1016/j.bbrc.2020.09.027

17. Zhao Q, Yang W-R, Wang X-H, et al. Clostridium butyricum alleviates intestinal low-grade inflammation in TNBS-induced irritable bowel syndrome in mice by regulating functional status of lamina propria dendritic cells. World J Gastroenterol. 2019;25:5469-5482. doi:10.3748/wjg.v25.i36.5469
18. He Z, Sun X, Ma Z, et al. Heat shock protein 70 protects mouse against post-infection irritable bowel syndrome via up-regulating intestinal gammadelta $\mathrm{T}$ cell's Th17 response. Cell Biosci. 2018;8:38. doi:10.1186/s13578-018-0237-z

19. Beattie L, Sawtell A, Mann J, et al. Bone marrow-derived and resident liver macrophages display unique transcriptomic signatures but similar biological functions. $J$ Hepatol. 2016;65:758-768. doi:10.1016/j.jhep.2016.05.037

20. Nielsen MC, Andersen MN, Moller HJ. Monocyte isolation techniques significantly impact the phenotype of both isolated monocytes and derived macrophages in vitro. Immunology. 2020;159:63-74. doi:10.1111/imm.13125

21. Tamoutounour S, Henri S, Lelouard H, et al. CD64 distinguishes macrophages from dendritic cells in the gut and reveals the Th1-inducing role of mesenteric lymph node macrophages during colitis. Eur J Immunol. 2012;42:3150-3166. doi:10.1002/ eji.201242847

22. He C, Yu T, Shi Y, et al. MicroRNA 301A Promotes Intestinal Inflammation and Colitis-Associated Cancer Development by Inhibiting BTG1. Gastroenterology. 2017;152(1434-1448):e1415. doi:10.1053/j.gastro.2017.01.049

23. Yang X, Liu H, Ye T, et al. AhR activation attenuates calcium oxalate nephrocalcinosis by diminishing M1 macrophage polarization and promoting M2 macrophage polarization. Theranostics. 2020;10:12011-12025. doi:10.7150/thno.51144

24. Black CJ, Yiannakou Y, Houghton LA, et al. Anxiety-related factors associated with symptom severity in irritable bowel syndrome. Neurogastroenterol Motil. 2020;32:e13872. doi:10.1111/nmo.13872

25. Zeinali M, Rezaee SA, Hosseinzadeh H. An overview on immunoregulatory and anti-inflammatory properties of chrysin and flavonoids substances. Biomed Pharmacother. 2017;92:998-1009. doi:10.1016/j. biopha.2017.06.003

26. Kim BH. Kurarinone regulates immune responses through regulation of the JAK/STAT and TCR-mediated signaling pathways. Biochem Pharmacol. 2013;85:1134-1144. doi:10.1016/j.bcp.2013.01.005

27. Murray IA, Patterson AD, Perdew GH. Aryl hydrocarbon receptor ligands in cancer: friend and foe. Nat Rev Cancer. 2014;14:801-814. doi:10.1038/nrc3846

28. Shinde R, Hezaveh K, Halaby MJ, et al. Apoptotic cell-induced AhR activity is required for immunological tolerance and suppression of systemic lupus erythematosus in mice and humans. Nat Immunol. 2018;19:571-582. doi:10.1038/s41590-018-0107-1
Journal of Inflammation Research

\section{Publish your work in this journal}

The Journal of Inflammation Research is an international, peerreviewed open-access journal that welcomes laboratory and clinical findings on the molecular basis, cell biology and pharmacology of inflammation including original research, reviews, symposium reports, hypothesis formation and commentaries on: acute/chronic inflammation; mediators of inflammation; cellular processes; molecular mechanisms; pharmacology and novel anti-inflammatory drugs; clinical conditions involving inflammation. The manuscript management system is completely online and includes a very quick and fair peerreview system. Visit http://www.dovepress.com/testimonials.php to read real quotes from published authors. 